\title{
ANÁLISIS DE LA ADAPTACIÓN DE LOS LIBROS DE TEXTO DE ESO AL CURRÍCULO OFICIAL, EN EL CAMPO DE LA QUÍMICA
}

\section{Calvo Pascual, M. Araceli y Martín Sánchez, Manuela}

Departamento de Didáctica de las Ciencias Experimentales. Facultad de Educación de la Universidad Complutense de Madrid. Centro de Formación del Profesorado macp@quimicosmadrid.org mmartins@edu.ucm.es diciex@eucmos.sim.ucm.es

Resumen. Este artículo muestra un análisis del tratamiento dado a la química en los libros de texto de ciencias de la ESO. Se hace una revisión de la bibliografía existente sobre la investigación del libro de texto y se elabora un instrumento de análisis para examinar diversos aspectos de las unidades de química de una muestra de libros de texto.

Los resultados obtenidos en este análisis indican hasta qué punto los libros de texto facilitan que los estudiantes consigan los objetivos generales presentes en la legislación oficial.

Palabras clave. Enseñanza de las ciencias, análisis de libros de texto de secundaria, currículo de ESO.

Summary. This paper shows an analysis of how chemistry is treated in secondary school textbooks. We have reviewed the existing bibliography on textbook research and have developed a tool to study different aspects of chemistry units from a sample of textbooks.

The results thus achieved show how far textbooks enable pupils to attain the general objectives required by official regulations.

Keywords. Sciences teaching, secondary science teaching, analisys of books for secondary science teaching.

\section{INTRODUCCIÓN}

Según la legislación vigente, el tratamiento dado a la química en la educación secundaria obligatoria debe determinar que los estudiantes, por una parte, adquieran la capacidad crítica de discernir lo correcto de lo erróneo en los temas sociales relacionados con la química $y$, por otra, que tengan la motivación necesaria para continuar estudiándola. Teniendo en cuenta que el desarrollo del currículo oficial depende en gran medida del libro de texto, ya que es utilizado por la mayor parte de los profesores como el recurso didáctico principal y, en muchas ocasiones, casi único, en nuestro trabajo nos planteamos la pregunta siguiente: ¿Los libros de texto, por el hecho de estar publicados, se adaptan adecuadamente al currículo? 
El procedimiento seguido para intentar contestar a esta pregunta ha sido comenzar estudiando la legislación oficial relativa a la enseñanza de la química en la educación secundaria obligatoria, la legislación relativa a la aceptación oficial de los libros de texto y las investigaciones que hemos encontrado en la bibliografía relacionadas con este tema. Tomando como base todos estos documentos se ha redactado una ficha y una plantilla que, después de validar con una pequeña muestra de libros de texto, se ha aplicado a los libros sobre los que se ha realizado la investigación. Con los datos obtenidos en la investigación se llega a unas conclusiones.

\section{Investigaciones relacionadas con el libro de texto}

El estudio bibliográfico que hemos hecho sobre investigaciones relacionadas con el libro de texto es tan extenso que es prácticamente imposible pretender incluirlo en un artículo, ni siquiera comentarlo; por eso nos vamos a limitar a citar las que nos han servido de pauta fundamental para el estudio empírico posterior y que agrupamos en los siguientes apartados: $a$ ) definiciones y características de los libros de texto; $b$ ) metodologías de análisis de libros de texto; $c$ ) aspectos a considerar en la elaboración y en el análisis de libros de texto; y $d$ ) modificaciones introducidas en los sucesivos cambios curriculares.

\section{Definiciones y características de los libros de texto}

El Diccionario de Ciencias de la Educación (Mesanza, 1983) define el libro de texto como «el recurso didáctico que ofrece al alumno la información relevante de un nivel, curso o disciplina sistematizada y adecuada al currículo en el que se inscribe».

Selander (1995) afirma que el libro de texto es el material básico para la clase y la herramienta más genuina para el profesor y para el alumno, no es ni más ni menos que la casi totalidad del currículo.

Navarro (1985) lo define como «aquel recurso técnicoeducativo, legalmente reconocido, que abre al usuario a la realidad cultural, científica y social-personal de su tiempo».

En palabras de Richaudeau (1981), «el manual escolar es un material impreso, estructurado, destinado a utilizarse en un determinado proceso de aprendizaje y formación».

En cuanto a las características del libro de texto destacaríamos el trabajo de Marchesi y Martín (1991) que, entre otros muchos criterios, opinan que debe ofrecer a los profesores vías de análisis y reflexión para que puedan adaptarlos con más facilidad a las condiciones sociales y culturales en las que van a desarrollar su trabajo. No deben ser propuestas cerradas e inflexibles. Incluirán tres tipos de contenidos, que deben trabajarse interrelacionadamente. Tendrán un amplia gama de actividades didácticas, ayudarán a consolidar la organización curricular, atenderán a los temas transversales y señalarán los criterios de evaluación.
Johnsen (1996), refiriéndose al lenguaje, indica que cada materia debe tener un lenguaje ordinario y un lenguaje para propósitos especiales, que sea capaz de inspirar a la mayoría de los alumnos el deseo de leer y usar tal lenguaje.

Para DeBolt (1992), los profesores deben buscar libros que sean flexibles, estén escritos claramente, sean interactivos y atractivos, estén integrados en el currículo e incluyan modos alternativos de explicar los conceptos esenciales y generalizaciones; los mapas, gráficos, tablas e imágenes deben estar integrados con la lectura.

Según Prats (1997) es importante que: contengan la explicitación de sus finalidades didácticas, sean capaces de motivar a los alumnos, traten correctamente la información, permitan estructurar la actividad de la clase y promuevan el crecimiento del conocimiento.

\section{Metodologías de análisis de libros de texto}

La mayoría de las metodologías empleadas para analizar los libros de texto utilizan la técnica de análisis de contenido, que puede definirse como: el método consistente en considerar todo mensaje como una secuencia de elementos aislables, susceptibles de ser ordenados por categorías y tratados de manera estadística (Rodríguez, Escudero y Bolívar, 1978); el análisis cuantitativo sistemático de materiales de enseñanza (Tamir y García, 1992); la evaluación de un cuerpo de material de comunicación (libros de texto) para determinar su significado (Jeffery y Roach, 1994); o, como dicen Krippendorf y Wandersee (citados por los anteriores) la aplicación de un esquema de clasificación al material analizado con respecto a un contenido de interés.

A su vez este análisis de contenido puede tener distintos enfoques y propósitos: el análisis de la estructura sintáctica, semántica, simbólica, curricular, secuencial, evolutiva o del grado de dificultad de los contenidos (Jiménez y Perales, 2001).

Eltinge y Roberts (1993) hablan del análisis de contenido linguiístico.

Sánchez (1997) comenta los dos tipos de organización del contenido que suelen presentar los libros de texto: lineal y espiral, así como los criterios a tener en cuenta a la hora de tomar decisiones sobre el tipo de recursos y las estrategias a emplear, criterios que también indica Navarro (1985).

Otros muchos métodos aparecen descritos en la bibliografía (asociativo, hermenéutico, de comprensión lectora, análisis ideológico, etc.), que no incluimos por problemas de espacio.

\section{Aspectos a considerar en la elaboración y en el análisis de libros de texto}

El Diccionario de las Ciencias de la Educación (Mesanza, 1983) resume las fases que deben tenerse en cuenta al hacer un libro de texto, y de ellas tomamos las que deberían va- 
lorarse también al analizarlo: son un número considerable de aspectos que a su vez son los que aparecen también, aunque de forma más detallada, en otras citas bibliográficas (Heredia, 1983; López, 1986; Fernández, 1989). Los especificamos a continuación: $a$ ) determinación de objetivos, siguiendo lo que se indica en los cuestionarios y programas oficiales; $b$ ) atención a la psicología del alumno a quien se dirige (intereses, actitudes, aptitudes); c) atención a la estructura de la materia; y $d$ ) las leyes de aprendizaje.

López (1986) y Beas (1999) se refieren a otros aspectos como tipografía, organización del texto, tamaño del libro, etc.

Son muchos los cuestionarios encontrados en la bibliografía, y todos ellos pueden complementarse entre sí, pero destacaremos el de Richaudeau (1981), que dice que se deben valorar bajo cuatro aspectos: contenido (calidad, cantidad y exactitud de la información), comunicación (legibilidad y densidad), método (organización y adaptabilidad) y objeto material (solidez, manejabilidad y costo). Para ello plantea 216 preguntas con varios tipos de indicadores cada una.

Otros cuestionarios que estimamos que son muy interesantes son los de Estébanez (1981), Rosales (1983) y Fernández Uría (1978), que, aunque hayan sido elaborados para otro tipo de currículos, por su riqueza son totalmente válidos para estudios actuales aplicados a la química.

Dentro de los cuestionarios más actuales, merecen especial mención, entre todos los encontrados en la bibliografía, los de Strube (1989) y Chiapetta, Sethna y Fillman (1991) sobre modelos para el análisis de libros de texto de física y química. Newton (1990) y Fetsko (1992) se refieren a las características y extensión de los contenidos. Parcerisa (1997) dice que el análisis debe tener en cuenta el proyecto curricular del centro. Gayán y García (1997) propone un cuestionario para valorar el material existente. Guisasola (1997) busca las visiones distorsionadas de la ciencia que aparecen en los libros. Furió (1997) valora las carencias epistemológicas. Guirao y Jaén (1997) analizan un tema de astronomía. De Posada (1999) estudia el tema de enlace metálico e Ibáñez (1999) intenta valorarlos desde las perspectivas del alumno, si realmente obliga a los alumnos a pensar.

Entre los cuestionarios específicos, caben destacar los relativos a: actividades en general (Santos, 1991; Islas y Guridi, 1999), actividades de lápiz y papel (Bullejos (1983; Perales y Martos, 1997), actividades experimentales (Tamir y García, 1992; Rivera e Izquierdo, 1996; Pérez y Fortes, 1997), ilustraciones (Jiménez y Perales, 1997) e ideas previas (Sanger y Greenbowe, 1999).

\section{Modificaciones introducidas con los sucesivos cambios curriculares}

En las investigaciones realizadas se deduce que existe una continuidad de los libros editados en los sucesivos cambios curriculares: los modelos e ideas anteriores si- guen perviviendo en los nuevos libros a pesar de lo que decida el currículo (Selander, 1995) y las variaciones mayores se refieren a la presentación del libro (Westbury, 1990; De Posada, 1999).

Según Prats (1997), la aplicación de la LOGSE no ha hecho que los libros dejen de ofrecer una secuencia cerrada y una batería estandarizada de actividades. El libro de texto se sigue concibiendo, en gran parte, como manual único y, en pocas ocasiones, incluye bibliografía o información complementaria. El concepto de ciclo realmente no ha sido asumido, excepto en algún caso aislado. Los aspectos mejor resueltos son los formales y el peor, la atención a la diversidad. En cuanto a las diferencias entre editoriales, la mayoría muestra una línea y unos planteamientos unificados en los distintos materiales que editan, aunque a veces se observan líneas distintas en una misma editorial entre los materiales de distinto ciclo (Parcerisa, 1997).

Algunas investigaciones como la de Jiménez y Perales (1997) admiten ligeras mejorías en los libros a lo largo de los años, sobre todo desde el punto de vista metodológico, y un posible aumento de las referencias dedicadas a temas de la vida cotidiana y ciencia-tecnología-sociedad. Perales y Martos (1997) admiten un notable avance en cuanto a la resolución de problemas en la enseñanzaaprendizaje de las ciencias; los textos LOGSE utilizan un mayor número de problemas cualitativos y abiertos. Se detecta también un incremento significativo de la manipulación de objetos reales y consulta de material de apoyo, aunque no es así en el suministro del algoritmo de resolución y en la resolución grupal, existiendo un incremento significativo en los procedimientos de resolución.

Jiménez y Perales (2001) consideran que hay dos líneas en los libros, la de corte más tradicional y la de los influenciados por tendencias asociadas al proceso de reforma de la educación secundaria (como pueda ser el «constructivismo» y el movimiento de las concepciones alternativas de los alumnos). Indican que se ha observado en los últimos años una transformación de libro «continente para el alumnado» al libro «guión para el profesorado», que tiene una creciente complejidad y no se limita a presentar de forma ordenada la información disciplinar sino que aspira a organizar el trabajo en el aula planteando preguntas a los alumnos y describiendo actividades o problemas que requieren la supervisión del profesorado.

\section{ESTUDIO EMPÍRICO}

\section{Objetivos}

El objetivo general de nuestro estudio empírico ha sido valorar en qué grado los distintos libros de texto del área de ciencias de la naturaleza de la educación secundaria obligatoria, publicados recientemente por editoriales de amplia difusión, se ajustan al currículo oficial en los temas correspondientes a química. 
Para ello se ha estudiado:

- Extensión de la disciplina de química.

- Secuenciación de la disciplina a lo largo de los dos ciclos de la ESO.

- Se han tratado de identificar y localizar en las distintas unidades los contenidos conceptuales, procedimentales y actitudinales descritos en el currículo oficial.

\section{Metodología}

Primero se llevó a cabo un análisis inicial con tres libros de física y química de $3^{\circ}$ de ESO, de 1995, de las editoriales Anaya, Santillana y SM para validar el instrumento de análisis diseñado, consistente en una ficha y una plantilla.

Tras esta validación y después de hacer los cambios necesarios, se examinó con el instrumento de análisis definitivo (Anexo I), una muestra de 34 libros de los cuatro cursos de la ESO de las editoriales Anaya, Edebé, Edelvives, McGrawHill, Santillana y SM (dos proyectos), que se numeran aleatoriamente para respetar la privacidad de los resultados concretos de cada libro (las referencias aparecen en el Anexo III).

De cada editorial se ha analizado la última edición existente en el mercado en el momento en que se realizó la investigación.

En el caso de las editoriales Anaya, Edebé, Edelvives y McGraw-Hill se dispuso del libro del profesor como apoyo al análisis del libro del estudiante.

Los bloques de contenidos analizados fueron el 1 y el 3 del currículo oficial del área de ciencias de la naturaleza de la educación secundaria obligatoria que corresponden a «Diversidad y unidad de estructura de la materia» y «Los cambios químicos».

\section{Resultados generales del análisis}

Importancia cuantitativa dada a la química respecto a los otros campos del área de ciencias de la naturaleza

Después de hacer un estudio del número de páginas y de unidades didácticas se comprueba que todas las editoriales conceden la mayor extensión a la química de $3^{\circ}$ de ESO, seguida de $4^{\circ}$ de ESO en cinco editoriales, y de $1^{\circ}$ de ESO en otras dos; en concreto, el orden decreciente de extensión dedicada a la química es el siguiente:

$3^{\circ}$ de $\mathrm{ESO}>4^{\circ}$ de $\mathrm{ESO}>2^{\circ}$ de $\mathrm{ESO}>1^{\circ}$ de ESO (editoriales I, IV y VI).

$3^{\circ}$ de $\mathrm{ESO}>4^{\circ}$ de $\mathrm{ESO}>1^{\circ}$ de $\mathrm{ESO}>2^{\circ}$ de ESO (editoriales II y VII).

$3^{\circ}$ de $\mathrm{ESO}>1^{\circ}$ de ESO $>4^{\circ}$ de ESO $>2^{\circ}$ de ESO (editorial III, con mayor núm. de págs.).

$3^{\circ}$ de ESO $>1^{\circ}$ de $\mathrm{ESO}>4^{\circ}$ de ESO $>$ en $2^{\circ}$ de ESO no aparece química (editorial $\mathrm{V}$ ).

\section{Características generales de los libros de texto analizados}

A continuación hacemos un resumen del estudio de las características más importantes:

\section{Presentación}

- Todos los libros de todas las editoriales tienen índice. - La mayor parte de las ilustraciones tienen texto explicativo.

- Para resaltar lo significativo se usan letras en negrita y recuadros en colores.

Estos tres aspectos favorecen que el libro de texto sea más útil y fácil de manejar en clase, y que el alumno/a pueda entender y retener mejor los contenidos fundamentales. En cambio:

- Solamente la editorial II tiene un glosario; las editoriales III y IV tienen láminas plastificadas, y la editorial III en primer ciclo y la IV en segundo ciclo tienen solucionario.

- Por otra parte, los títulos, en general, no son indicativos exactos del contenido de cada unidad, solamente las editoriales I y $\mathrm{V}$ tienen títulos adecuados.

\section{Estructura de las unidades didácticas}

Todas las unidades de los libros de cada editorial tienen la misma estructura general:

- Página inicial.

- Desarrollo teórico con actividades.

- Actividades y textos complementarios.

Tipos de actividades e ilustraciones

El gráfico siguiente muestra el número de los distintos tipos de actividades e ilustraciones que aparecen a lo largo de los cuatro cursos de la ESO.

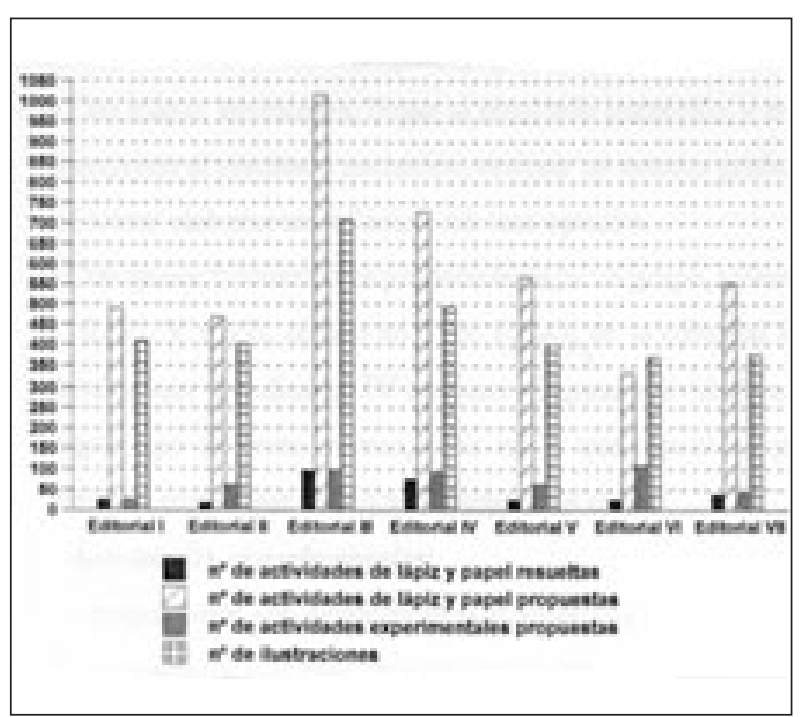


La editorial III es la que tiene mayor número de actividades de lápiz y papel propuestas, resueltas, y también de ilustraciones, siendo además, esta editorial, la segunda que tiene el mayor número de actividades experimentales, superada en este tipo de actividades sólo por la editorial VI.

Las actividades de lápiz y papel se nombran como $a c$ tividades, ejercicios o problemas, y en el caso de las experimentales se nombran como experiencias, salvo en el $2^{\circ}$ ciclo de la editorial IV, en la que se llaman experimentos, y en las editoriales VI y VII las denominan investigaciones.

Es decir, que en general los libros de texto no favorecen que el alumno/a distinga entre un ejercicio y un problema o entre una experiencia y un experimento.

\section{Características y funciones de las actividades e ilustra- ciones}

Actividades de lápiz y papel resueltas

En el primer ciclo, en concreto en $2^{\circ}$ de ESO, el mayor número de actividades son cuestiones en las que se justifica la respuesta.

En el $2^{\circ}$ ciclo y sobre todo en $3^{\circ}$ de ESO hay un mayor número de ejercicios cuantitativos, en los que se da más importancia a la utilización de las fórmulas y al cálculo que al análisis conceptual, cuando este análisis es fundamental para que estas actividades resueltas sean útiles para favorecer la autonomía del alumno/a.

\section{Actividades de lápiz y papel propuestas}

- El orden de frecuencia decreciente es el siguiente: cuestiones $>$ ejercicios cuantitativos $>$ ejercicios cualitativos $>$ problemas de investigación abiertos. Cabría citar que los ejercicios cualitativos aparecen en un mayor número de cursos, pero que problemas de investigación abiertos, que son un recurso muy completo para trabajar en la clase, sólo hemos encontrado uno en el primer ciclo de la editorial II y otro en $3^{\circ}$ de ESO de la editorial V.

- El mayor número de preguntas cuya respuesta está implícita en el texto aparece en la editorial III $\left(2^{\circ}, 3^{\circ}\right.$ y $\left.4^{\circ}\right)$, y explícita en la editorial VII $\left(1^{\circ}, 2^{\circ}\right.$ y $\left.3^{\circ}\right)$, y en el $2^{\circ}$ ciclo de la editorial V.

En las actividades de lápiz y papel, se pide:

- Con mayor frecuencia, llegar al resultado numérico y, en el caso de la editorial III, analizar e interpretar el resultado numérico, realizar un estudio gráfico, diseñar un experimento, y comentar un texto, elaborar un informe y dar una opinión.

- Con menor frecuencia se propone trabajar en grupo, solamente en un curso de cinco editoriales: I, II, III, V y VII, siendo en $2^{\circ}$ de ESO de la editorial III donde aparece más. Es importante resaltar que la editorial IV no tiene ninguna actividad en la que el alumno tenga que redactar algún tipo de texto escrito y es en la que menos se pide consultar bibliografía, aspectos fundamentales que deben trabajarse en la clase.

\section{Actividades experimentales}

La mayor parte son experimentos cualitativos.

Las características más importantes de las actividades experimentales son:

- En prácticamente todas las actividades se indica el procedimiento operativo que debe seguirse: aparece una ilustración con el montaje experimental y se especifica el resultado que va a obtenerse, es decir, responden a actividades «tipo receta».

- Lo normal es que se necesite emplear un material específico de laboratorio para su realización.

- Es poco frecuente que se den normas de seguridad, el mayor número aparece en el $2^{\circ}$ ciclo de las editoriales VI y III. Tampoco se suele pedir que tomen datos, se justifique lo que sucede, se realice un estudio gráfico, se diseñe un nuevo experimento, se elabore un informe o se trabaje en grupo, por lo que no permiten que el alumno/a conozca y se inicie en lo que es una verdadera investigación, siguiendo una metodología científica.

\section{Ilustraciones}

Predominan las fotografías, de las que un 56\% son motivadoras o decorativas, apareciendo el mayor porcentaje con esta finalidad en las editoriales VI y I ( $77 \%$ y $75 \%$ respectivamente) y el menor porcentaje en la editorial III (39\%).

Después de las fotografías, el mayor porcentaje de ilustraciones corresponde a los dibujos; la mayoría de ellos son para facilitar la comprensión de un concepto o actividad.

Es de destacar que hay muy pocas ilustraciones sobre científicos ilustres, que, en cambio, enriquecen el conocimiento del alumno y favorecen que contextualice la obra del científico con su época.

Las ilustraciones son tablas y gráficos, algunas son parte fundamental del desarrollo teórico pero otras son una información añadida aunque no necesaria para la comprensión del texto o forman parte del enunciado de actividades de lápiz y papel propuestas. También aparecen esquemas y mapas conceptuales relacionados con el desarrollo teórico, recurso útil tanto en clase como en el estudio individual del alumno/a.

\section{Contenidos del currículo oficial presentes}

- Todas las editoriales carecen de alguno de los contenidos especificados en el currículo oficial. Las editoriales III, IV y VI son las que tienen un mayor número de contenidos.

- Los contenidos que aparecen en mayor medida en los libros son los conceptuales, existiendo más carencias con relación 
a los contenidos procedimentales y los actitudinales, aunque los tres son importantes en el trabajo diario en clase.

- Entre los contenidos que aparecen con menor frecuencia citaremos, por considerarlos importantes, los siguientes: a) el contenido procedimental relativo a la estimación del error cometido en el manejo de instrumentos de medida, y los actitudinales: «sensibilidad por el orden y la limpieza del lugar de trabajo y del material utilizado»; y $b$ ) «análisis de las medidas internacionales que se establecen al respecto del efecto de los productos químicos presentes en el entorno sobre la salud, la calidad de vida, el patrimonio artístico y el futuro de nuestro planeta».

- Nos parece importante destacar algo muy positivo: «la identificación de sustancias importantes por su utilización en la vida diaria» se encuentra en todos los cursos de todas las editoriales.

\section{Secuenciación}

No hay gran diferencia con relación a la secuenciación entre editoriales. Teniendo en cuenta el conjunto de los cuatro cursos, se puede decir que la secuencia general es la siguiente:

Propiedades de la materia, estados físicos y cambios de estado - Clasificación de la materia, concentración de las disoluciones y métodos de separación de mezclas (Se inicia en $1^{\circ}$ )

\section{Átomo $\left(\right.$ Se inicia en $\left.2^{\circ}\right)$}

Sistema periódico- Enlace - Formulación y nomenclatura de química inorgánica (Se inicia en $3^{\circ}$ )

Reacciones químicas (Se inicia en $2^{\circ}$ )

Leyes ponderales y volumétricas - Cálculos estequiométricos Termodinámica - Cinética -

Química industrial (Se inicia en $3^{\circ}$ )

Química orgánica- Bioquímica (Se inicia en $4^{\circ}$ )

Los contenidos del bloque 1 «Diversidad y unidad de la estructura de la materia» del currículo oficial suelen aparecer en el primer ciclo y vuelven a estudiarse en el segundo ciclo, sobre todo en $3^{\circ}$ de ESO, donde se estudia, el enlace y las reglas de formulación y nomenclatura inorgánica. Los contenidos correspondientes al bloque 3 «Los cambios químicos» se estudian prioritariamente en el segundo ciclo.

\section{Otros aspectos}

\section{Ideas previas}

Con el fin de detectar las ideas previas se proponen una serie de cuestiones al comienzo de cada unidad.

\section{Temas transversales}

Los temas transversales que aparecen con más frecuencia son:
«Educación ambiental», «Educación del consumidor»y «Educación para la salud», con unidades específicas en la editorial III, en $1^{\circ}$ y $2^{\circ}$, en la IV en $3^{\circ}$ y en la $V$ en $1^{\circ}$.

La «Educación vial» y la «Educación para la igualdad de oportunidades de ambos sexos» aparecen de forma muy escueta. Únicamente en la editorial I aparecen estos temas independientes de la unidad y los pone al final de la mis$\mathrm{ma}$; en el resto de las editoriales aparecen a lo largo de la unidad en las editoriales II, VI y VII, o incluidos durante el desarrollo y además al final en las editoriales III, IV y V.

\section{Interdisciplinariedad}

Únicamente puede considerarse interdisciplinar una unidad de $1^{\circ}$ de la editorial III y una de $4^{\circ}$ de la editorial IV. En las restantes a veces hay explicaciones o ilustraciones en las que se relacionan los contenidos que se están explicando con geología, biología, física, geografía, historia y arte. Con relación a lengua se puede encontrar citado el origen de las palabras y, en conexión con literatura, existen fragmentos de libros de divulgación científica o artículos. En $3^{\circ}$ de la editorial $\mathrm{V}$ aparecen fragmentos de dos obras literarias.

\section{Conexión entre los distintas temas}

La conexión se limita a la aparición de comentarios, seguidos de actividades, frecuentemente al margen, sobre contenidos que el alumnado debe conocer, generalmente conceptos vistos ese año en unidades anteriores. La editorial VII es la que presenta más actividades de este tipo (en $3^{\circ}$ ) y la que más se refiere a contenidos procedimentales.

\section{Analogías}

Aparecen tanto analogías útiles para los alumnos/as -porque facilitan la comprensión de conceptos, generalmente conceptos microscópicos, comparándolos explícitamente con objetos macroscópicos conocidos por el alumno/a-, como otras carentes de utilidad, que pueden inducir a errores conceptuales o dificultar la utilización correcta del vocabulario científico (principalmente en las editoriales III y IV).

En $4^{\circ}$ de ESO es donde menos analogías se emplean, quizás porque se considera que el alumno ya tiene un mayor conocimiento del área y no necesita tanto este recurso.

\section{Referencias históricas}

Aun siendo importantes por favorecer la interdisciplinariedad y permitir contextualizar los contenidos tratados, se limitan a una ubicación en el siglo o año de un descubrimiento, fechas de nacimiento y muerte de científicos destacados, seguidas de biografías no muy extensas de científicos conocidos; únicamente en las editoriales V y VII tienen más extensión.

La tabla I muestra las editoriales destacables en los aspectos señalados. 
Tabla I

\begin{tabular}{|c|c|c|}
\hline & Editoriales en las que aparecen más & Datos destacables \\
\hline Ideas previas & $\begin{array}{l}\text { En todas las unidades de las editoriales I y II, seguida de la } \\
\text { editorial IV. }\end{array}$ & $\begin{array}{l}\text { - En las editoriales III y VI las ideas previas } \\
\text { se intentan detectar sólo en } 1^{\circ}, 2^{\circ} \text { y } 3^{\circ} \text {, } \\
\text { disminuyendo al avanzar en los cursos. } \\
\text { - La editorial V es la única que las intenta } \\
\text { detectar en relación con los tres tipos de } \\
\text { contenidos (el resto conceptuales, seguidos de } \\
\text { procedimentales). }\end{array}$ \\
\hline Temas transversales & $\begin{array}{l}\text { En la editorial III, seguida de la VI, siendo esta última la } \\
\text { que los trata más regularmente. }\end{array}$ & $\begin{array}{l}\text { La editorial VII es la que menos se ocupa de } \\
\text { ellos. }\end{array}$ \\
\hline Interdisciplinariedad & Editorial III. & $\begin{array}{l}\text { La que menos la tiene en cuenta es la editorial } \\
\text { V seguida de la VII. }\end{array}$ \\
\hline $\begin{array}{l}\text { Conexión entre los distintos } \\
\text { temas }\end{array}$ & $\begin{array}{l}\text { La editorial III es la que más homogéneamente conecta a } \\
\text { lo largo de los cursos los distintos temas entre sí. }\end{array}$ & $\begin{array}{l}\text { La editorial VI es la que establece una menor } \\
\text { conexión entre los contenidos de las distintas } \\
\text { unidades o de los distintos cursos. }\end{array}$ \\
\hline Analogías & En las editoriales III y IV. & Aparecen menos analogías en la editorial VII. \\
\hline Referencias históricas & En la editorial VII, seguida de la editorial V. & $\begin{array}{l}\text { En } 3^{\circ} \text { de ESO de la editorial III es destacable } \\
\text { el desarrollo histórico de la industria química. }\end{array}$ \\
\hline
\end{tabular}

\section{Inexactitudes y errores detectados}

El orden de frecuencia decreciente de errores detectados es el siguiente:

Inexactitudes o imprecisiones > Errores químicos > Errores lingüísticos

- No hay grandes diferencias entre editoriales excepto la editorial II, que tiene bastantes errores lingüísticos (de acentuación), en el primer ciclo, y errores de edición (el símbolo de varios elementos) en el segundo ciclo. Teniendo en cuenta la dificultad que muchos alumnos tienen, tanto para escribir como para formular correctamente, es muy importante que los libros de texto no tengan este tipo de errores, que favorecen confundirlos aún más.

- Son también destacables, por la frecuencia con la que aparecen, las inexactitudes, imprecisiones y errores químicos debidos a que los libros no están convenientemente actualizados, sobre todo en relación con las normas dadas por la IUPAC sobre nomenclatura y simbología. En concreto, todas las editoriales excepto la I, usan nomenclaturas no admitidas actualmente por la IUPAC (si bien la editorial $\mathrm{V}$ sólo lo hace en $1^{\circ} \mathrm{y}$ las editoriales III y IV especifican que están desaconsejadas). Las editoriales I, V y VII sólo emplean la nomenclatura sistemática en los compuestos binarios y, en general, se emplea poco esta nomenclatura respecto a las demás.

- Otros ejemplos de inexactitudes e imprecisiones son:

Ilustraciones que no guardan una escala adecuada, por ejemplo, dibujan las partículas del átomo del mismo tamaño que el átomo, moléculas del tamaño de los hombres (editoriales II y IV), o dibujos sobre reacciones químicas en las que no se conserva el color de cada átomo (editorial II).

Hablan del uso actual de los CFC en determinados aparatos y recipientes cuando hace tiempo que está prohibida su utilización (editorial I), utilizan la polémica regla de tres (editoriales I y III), dan las unidades de la masa molecular como $\mathrm{g} / \mathrm{mol}$ (editoriales III y IV) o bien utilizan expresiones en desuso, como, por ejemplo, representar la molaridad de un compuesto simbolizando su fórmula entre corchetes (editorial IV) o citar la palabra electropositividad (editorial V).

\section{- Ejemplos de errores químicos son:}

En ninguna editorial aparece el sistema periódico con todos los elementos existentes actualmente, siendo frecuente que los últimos elementos que se especifican no se nombren con los nombres actuales indicados por la IUPAC (editoriales I, II, V, VI, VII). De la misma forma, se numeran los grupos del sistema periódico con la nomenclatura antigua (editoriales II, IV, V y VII, aunque en estas dos últimas editoriales el texto sí cita cómo deberían numerarse actualmente).

Otros errores frecuentes relacionados también con las formulación y la nomenclatura inorgánica son: dar fórmulas con posiciones de aniones y cationes incorrectas (editoriales III, IV), o reglas para formular compuestos desfasadas y sin sentido químico (editoriales III, IV).

Se detecta también la utilización de nombres incorrectos, como, por ejemplo: anión hidroxilo (editoriales I y V), anión oxígeno (editorial I), anión flúor (editorial II) o bien nombrar los aniones tanto correctamente como in- 
correctamente: aniones cloro o cloruro, oxígeno u óxido, fósforo o fosfuro, carbono o carburo (editorial VII).

Aparecen también conceptos en desuso, como el de átomo-gramo (editorial V).

Un error destacable es representar el litro con $l$, en lugar de $L$, que no solamente aparece en las editoriales I, II, III, IV y V, sino que figura en el propio currículo oficial del BOE (como también aparece $g r$ para indicar el gramo y, sin embargo, los libros de texto analizados usan el símbolo correcto).

También se han observado en varias editoriales errores relativos a la clasificación de la materia: no se distingue claramente entre sustancia pura y mezcla (editoriales II, V, VI), o entre los distintos tipos de mezclas (I, II, V, VI). En realidad tampoco el currículo oficial es exacto en este aspecto, ya que uno de los contenidos conceptuales es «disoluciones, sustancias puras y elementos químicos», cuando los elementos son un tipo de sustancias puras.

Se han detectado errores conceptuales en relación con la molécula (la editorial V incluye el concepto dentro de los postulados de Dalton), no se distingue entre proceso exotérmico y espontáneo (editorial VI) y se cita la ley de las proporciones definidas como basada en la teoría atómica de Dalton (editorial VI).

Aparecen también errores a nivel procedimental, en la utilización del material de laboratorio (editorial III), o en ilustraciones en las que el montaje experimental no se ha realizado correctamente (editorial II), lo que favorece la confusión del alumno.

\section{Consecución de los objetivos generales de etapa y de área con el desarrollo curricular de los libros de texto de las distintas editoriales}

El análisis de los resultados obtenidos, teniendo en cuenta exclusivamente el libro de texto, concluye que ninguna de las editoriales analizadas permitiría que el alumnado consiguiera todos los objetivos marcados (detallados en el Real Decreto 1345/ 1991, de 6 de septiembre [BOE 13-9-91]) si solamente se toma como referencia el libro de texto.

- El grado de consecución de los objetivos respecto a los conceptos es mucho mayor que respecto a los procedimientos o las actitudes; los métodos procedimentales son los peor tratados.

Los aspectos más destacados son los siguientes:

- Es difícil que se alcance el objetivo relativo a la comprensión y producción de mensajes orales y escritos, o se alcanzará con muchas limitaciones, sobre todo en el aspecto de la expresión, importante para organizar y afianzar lo aprendido, porque las actividades se limitan a cuestiones cuya respuesta puede estar explícita en el texto, con lo cual el alumno/a copia el párrafo sin más. Los casos en que la contestación está implícita permiten desarrollar más la capacidad de comprensión, pero no la expresión, ya que pueden contestarse copiando la redacción del texto.

Están presentes con poca frecuencia y mal distribuidas a, lo largo de los cursos, actividades en las que los alumnos/ as tengan que comentar textos que tendrían que haberlos comprendido previamente, elaborar informes u opinar expresándose con sus propias palabras. Este tipo de actividades aparecen en mayor número en la editorial III.

En cuanto a los sistemas de notación y representación, se asocia el nombre de las sustancias más importantes con su fórmula, pero no hay suficientes actividades específicas que permitan que el alumno/a sepa comprender y utilizar este «lenguaje químico» en distintas situaciones. En este aspecto la mejor es la editorial VII.

- Sobre el objetivo obtención y selección de la información utilizando distintas fuentes, la mayor parte de las actividades se limitan a necesitar el propio libro como única fuente de información; en el caso de aparecer fragmentos extraídos de textos de la literatura científica, han sido previamente seleccionados artículos de revistas o periódicos -lo que sucede con poca frecuencia.

Donde hay más actividades de búsqueda de información es en la editorial III en $1^{\circ}$ y $3^{\circ}$.

Ningún libro propone bibliografía cuya lectura o consulta pueda ser interesante.

Solamente en $1^{\circ}$ y $2^{\circ}$ de la editorial IV se hace alusión a la búsqueda de información mediante las nuevas tecnologías.

- En relación con elaborar estrategias de identificación y resolución de problemas, no se plantean verdaderos problemas en los que haya que seguir el proceso lógico de resolución de problemas basándose en una metodología científica, ya que las actividades resueltas muestran la respuesta correcta o, todo lo más, justifican dicha respuesta y, si hay que utilizar fórmulas, dan más importancia a la utilización de dichas fórmulas y al cálculo que al análisis conceptual. En las actividades propuestas es poco frecuente tener que justificar la respuesta o analizar e interpretar ese resultado (siendo en la editorial III donde más aparecen).

Únicamente hay un problema de investigación abierto en $1^{\circ}$ y otro en $2^{\circ}$, de la editorial II, y uno en $3^{\circ}$, de la editorial V.

En los experimentos se explica todo lo que debe hacerse, y se da el resultado. Solamente en la editorial III es donde más hay que analizarlo e interpretarlo.

- Que el alumno/a pueda formarse una imagen ajustada de sí mismo y desarrollar actividades de forma autónoma depende, en gran medida, de la atención a la diversidad que concedan los libros de texto: pues bien, esta atención es más teórica que real, ya que, aunque en principio, al leer los libros, cinco editoriales (de la I a la V) presentan actividades clasificadas en función del grado de dificultad, no están claramente diferenciadas. En la misma línea, solamente dos textos complementarios de la editorial $\mathrm{V}$, considerados como atención a la diversidad, pueden considerarse con esta función y están concebidos para alumnos más aventajados.

En relación con la autonomía del alumno, los textos presentan actividades resueltas (el mayor número aparece en $3^{\circ}$ de la editorial III) y, en dos editoriales, hay actividades de autoevaluación tipo test: en el primer ciclo de la editorial III y en el segundo ciclo de la editorial IV. 
- En muy pocas ocasiones se propone el trabajo en grupo; en concreto, el mayor número de veces son cuatro, en $2^{\circ}$ de la editorial III.

- La implicación del ser humano en el medio ambiente se trata en todas las editoriales, salvo lo relativo al análisis de las medidas internacionales que se establecen sobre el efecto de los productos químicos presentes en el entorno sobre la salud, la calidad de vida, el patrimonio artístico $\mathrm{y}$ el futuro de nuestro planeta, que se tiene en cuenta en $3^{\circ}$ de las editoriales II, III y V.

- Las creencias populares, muchas veces erróneas, sobre aspectos científicos, así como la actitud de la sociedad en el desarrollo de la química, no aparecen reflejados en los libros de texto analizados.

- En todos los cursos de todas las editoriales se hace alusión a la relación de los temas explicados con situaciones de la vida cotidiana, permitiendo al alumno/a comprobar la importancia de la química en nuestra vida y en la sociedad.

Todas las editoriales hacen referencia a los avances científicos y tecnológicos que han supuesto o pueden suponer mejoras en la sociedad, al tratar los temas transversales de educación para la salud, educación del consumidor y educación ambiental; pero en muy pocas ocasiones se citan los avances acontecidos en momentos históricos anteriores al nuestro, con la época en la que tuvieron lugar, y sus características sociales, económicas o políticas, con lo que el alumno/a no es capaz de comprender la incidencia real que supusieron (excepto en $3^{\circ}$ de la editorial III ).

- Los libros de texto analizados no permiten conocer el patrimonio cultural relativo a la contribución científica española y su colaboración con otros países.

- Aunque, como ya se ha indicado, todas las editoriales tienen en cuenta los aspectos de la vida diaria desde la química, sobre todo en lo que a nutrición y hábitos de vida se refiere, integrados en los temas transversales de educación para la salud, educación del consumidor y educación ambiental, los aspectos estrictamente científicos relacionados con la adquisición de hábitos adecuados de higiene y seguridad en el trabajo son insuficientes -ya que el contenido actitudinal «Sensibilidad por el orden y la limpieza del lugar de trabajo y del material utilizado» es uno de los que menos aparece en los libros de texto (sólo en las editoriales II, V, VI y VII)-, y se plantean pocas normas de seguridad (el mayor número en las editoriales VI y III), fundamentales en el trabajo en el laboratorio.

\section{CONCLUSIONES}

En relación con la pregunta hecha al principio, que era el objetivo principal de esta investigación ¿Los libros de texto, por el hecho de estar publicados, se adaptan adecuadamente al currículo?, podemos concluir, teniendo en cuenta los resultados anteriores, que ninguna de las editoriales analizadas se adecua íntegramente al currícu- lo oficial, y, por lo tanto, no permite la consecución de todos los objetivos, fundamentalmente por la deficiente metodología utilizada para aplicar los procedimientos, pero también porque hay carencias relativas a los contenidos.

El Real Decreto 1345/1991, de 6 de septiembre (BOE 139-91), por el que se establece el currículo de la enseñanza secundaria obligatoria, cita que debe seguirse una metodología constructivista. En este sentido podríamos decir que los libros de texto analizados consideran las ideas previas de los alumnos/as, con las salvedades expuestas, pero se comprueba una deficiencia considerable en todas las editoriales si consideramos que el Real Decreto dice : «Los alumnos han de conocer y utilizar algunos métodos habituales en la actividad científica a lo largo del proceso investigador: planteamiento de problemas y formulación clara de los mismos; utilización de fuentes de información de manera sistemática y organizada; formulación de hipótesis pertinentes a los problemas; contraste de hipótesis mediante la observación rigurosa y, en ciertos casos, la planificación y realización de experimentos; recogida, organización y análisis de los datos; discusión de conclusiones y comunicación de resultados mediante el oportuno informe.»

La comparación de los resultados de esta investigación, con los obtenidos en trabajos anteriores encontrados en la bibliografía, demuestran que, desde que se ha implantado la LOGSE, las sucesivas ediciones que cada editorial ha ido haciendo de sus libros de texto no han tenido grandes diferencias en lo relativo al trato desigual que se da a los conceptos respecto a los procedimientos y las actitudes, al modo de plantear las actividades que los alumnos/as deben llevar a cabo, a la falta del trabajo en grupo o a la atención a la diversidad, siendo aspectos que deben mejorarse.

Además, los libros de texto presentan numerosas inexactitudes y errores químicos, muchos de ellos debidos a que, a pesar de ser ediciones modernas, determinados contenidos no han sido convenientemente actualizados.

En cambio, sí se observa, en los libros, una preocupación por el tratamiento de los temas transversales relativos a la educación ambiental, la educación para la salud y la educación del consumidor, así como un interés por mostrar la importancia que la química tiene en nuestra vida y en la sociedad.

Aunque lo dicho anteriormente pueda generalizarse a todas las editoriales analizadas, hay diferencias entre ellas, destacando unas u otras en función de los aspectos que se consideren, aunque la que mejor trata varios de los aspectos estudiados es la editorial III.

Es decir, que, considerando solamente el libro de texto, no se garantiza la homogeneidad de contenidos mínimos alcanzados por los alumnos/as para poder obtener un título de validez general.

Es obvio que es necesaria una adecuada labor docente para conseguir que los alumnos consigan los objetivos, 
con las deficiencias observadas. No es justificable que las editoriales «vendan» los libros de texto a los profesores diciendo que de esta forma se adecuan fielmente a la LOGSE.

Esto lleva a una doble recomendación:

- La primera a los docentes: es evidente que el libro de texto puede ser un recurso útil, pero no el único, sino un recurso más; algo que, si bien se ve como obvio, desde el punto de vista práctico debería tenerse mucho más en cuenta y facilitar, a los alumnos, otros recursos y medios para poderse formar.

Las deficiencias observadas sólo pueden suplirse con el trabajo diario de los profesores/as con los alumnos/as, con la continua autoevaluación para ir mejorando la metodología empleada, con el uso de los recursos más adecuados en función del momento y del alumno/a.

- La segunda recomendación es para las editoriales y se refiere a la importancia de facilitar materiales útiles y adecuados no sólo en la forma sino también en el fondo, pues, aunque el libro de texto no deba ser el único recurso, en niveles de secundaria, generalmente, se obliga a los alumnos a tenerlo y consultarlo, por lo que llega a ser el referente principal, ya no sólo durante las clases sino más aún en su trabajo individual en casa, donde refuerzan los conocimientos adquiridos. En definitiva su influencia sobre los alumnos/as es tan grande que se debe exigir una gran calidad en todos los aspectos.

\section{REFERENCIAS BIBLIOGRÁFICAS}

ABRAHAM, M. R., GRZYBOWSKI, E. B., RENNER, J. W. y MAREK, E. A. (1992). Understandings and misunderstandings of eighth graders of five chemistry concepts found in textbooks. Journal of Research in Science Teaching, 29(2), pp. $105-120$.

BEAS MIRANDA, M. (1999). Los libros de texto y las comunidades autónomas: una pesada torre de Babel. Revista Complutense de Educación, 10(2), pp. 29-52.

BULLEJOS DE LA HIGUERA, J. (1983). Análisis de actividades en textos de física y química en $2^{\circ}$ de BUP. Enseñanza de las Ciencias, 1, pp. 147-157.

CHIAPETTA, E. L., SETHNA, G. H. y FILLMAN, D. A. (1991). A quantitative analysis of high school chemistry textbooks for scientific literacy themes and expository learning aids. Journal of Research in Science Teaching, 28(10), pp. 939-951.

DEBOLT, G. P. (1992). Consumers of Textbooks: Concerns from the Classroom, en Herlihy, J. G. (ed.). The Textbook Controversy: Issues, Aspects and Perspectives, pp. 137-145. Norwood: Ablex Publishing Corporation.

ELTINGE, E. M. y ROBERTS, C. W. (1993). Linguistic content analysis: A method to measure science as inquiry in textbooks. Journal of Research in Science Teaching, 30(1), pp. 65-83.

ESTÉBANEZ ÁLVAREZ, J. et al. (1981). Análisis de los libros de texto de EGB. Boletín de la Real Sociedad Geográfica, 117, pp. 76-80, en Estébanez Álvarez, J. (1998). Obra dispersa. Tomo III, pp. 28-31. Madrid: Universidad Complutense de Madrid.

FERNÁNDEZ, M. (1989). El libro de texto en el desarrollo del currículo. Cuadernos de Pedagogía, 168, pp. 56-59.

FERNÁNDEZ URÍA, E. (1978). Análisis de libros de texto de ciencias en el bachillerato. Revista de Bachillerato, 6, pp. 10-14.
FETSKO, W. J. (1992). Approaching Textbook Selection Systematically, en Herlihy, J. G. (ed.). The Textbook Controversy: Issues, Aspects and Perspectives, pp. 129135. Norwood: Ablex Publishing Corporation.

FURIÓ, C. y GUISASOLA, J. (1997). Deficiencias epistemológicas en la enseñanza habitual de los conceptos de campo y potencial eléctrico. Enseñanza de las Ciencias, 15(2), pp. 259-271.

GAYÁN, E. y GARCÍA, P. (1997). ¿Cómo escoger un libro de texto? Desarrollo de un instrumento para evaluar los libros de texto de ciencias experimentales. Enseñanza de las Ciencias, núm. extra. V Congreso, pp. 249-250.

GIMENO SACRISTÁN, J. (1991). Los materiales y la enseñanza. Cuadernos de Pedagogía, 194, pp. 10-15.

GUIRAO, B. y JAÉN, M. (1997). ¿Cómo explican las estaciones los libros de texto de la ESO? Enseñanza de las Ciencias, núm. extra. V Congreso, pp. 251-252.

GUISASOLA, J. (1997). El trabajo científico y las tareas en la electrostática en textos de bachillerato. Alambique, 11, pp. $45-54$.

HEREDIA ANCONA, B. (1983). Manual para la elaboración de material didáctico. México: Trillas.

IBÁÑEZ, A. (1999). ¡Cuántos libros! ¿Cuál elijo? Boletín CDL. Especial libros de texto, 105, pp. 4-6.

ISLAS, S. M. y GURIDI, V. M. (1999). El quehacer científico versus el quehacer aúlico. Buscando rasgos del quehacer científico en libros de texto. Enseñanza de las Ciencias, 17(2), pp. 281-290.

JEFFERY, K. R. y ROACH, L. E. (1994). A study of the presence of evolutionary protoconcepts in pre-high school textbooks. Journal of Research in Science Teaching, 31(5), pp. 507-518. 
JOHNSEN, E. B. (1996). Libros de texto en el caleidoscopio. Estudio crítico de la literatura y la investigación sobre los textos escolares. Barcelona: Ediciones Pomares-Corredor.

JIMÉNEZ VALLADARES, J. y PERALES PALACIOS, F. J. (1997). Propuesta taxonómica para un análisis de las ilustraciones en los textos de física y química, en Jiménez Pérez, R. y Wamba Aguado, A. M. (eds.). Avances en la didáctica de las ciencias experimentales, pp. 519-528. Huelva: Universidad de Huelva.

JIMÉNEZ VALLADARES, J. D. y PERALES PALACIOS, F. J. (2001). Aplicación del análisis secuencial al estudio del texto escrito e ilustraciones de los libros de física y química de la ESO. Enseñanza de las Ciencias, 19(1), pp. 3-19.

LÓPEZ RODRÍGUEZ, N. (1986). Cómo valorar textos escolares. Madrid: Cincel-Kapelusz.

MARCHESI, A. y MARTÍN, E. (1991). Lo que dice el MEC sobre materiales. Cuadernos de Pedagogía, 194, pp. 46-48.

MESANZA LÓPEZ, J. (coord.) (1983). Diccionario de las Ciencias de la Educación. Vol. II. Publicaciones Diagonal Santillana para profesores. Madrid: Santillana.

MEYER, L. A. (1994 ). Los libros de texto de ciencias, ¿son comprensibles?, en Santa, C. M. y Alvermann, D. E. (comps.). Una didáctica de las ciencias. Procesos y aplicaciones, pp. 70-87. Buenos Aires: Aique.

NAVARRO LÓPEZ, J. (1985). «Evaluación de textos escolares». Tesis doctoral. Universidad Complutense de Madrid.

NEWTON, D. P. (1990). Teaching with text. Choosing, preparing and using textual materials for instruction. Londres: Kogan Page.

PARCERISA ARAN, A. (1997). Materiales curriculares. Cómo elaborarlos, seleccionarlos y usarlos. Barcelona: Graó.

PERALES PALACIOS, F. J. y MARTOS MEJÍA, F. (1997). Problemas tradicionales - problemas LOGSE: ¿algún cambio sustancial?, en Jiménez Pérez, R. y Wamba Aguado, A. M. (eds.). Avances en la didáctica de las ciencias experimentales, pp. 511-518. Huelva: Universidad de Huelva.

PÉREZ PLA, J. F. y FORTES DEL VALLE, M. C. (1997). La actividad experimental como estrategia de enseñanza recíproca. Un estudio de las propuestas que presentan los libros de texto de tercer ciclo de primaria y primer ciclo de ESO. Enseñanza de las Ciencias, núm. extra. V Congreso, pp. 267-268.

DE POSADA, J. M. (1999). The presentation of metallic bonding in high school science textbooks during three decades: science educational reforms and substantive changes of tendencies. Science Education, 83(4), pp. 423- 447.
PRATS, J. (1997). El nuevo modelo curricular y la elección de libros de texto, en Arranz Márquez, L. (coord.). Actas del V Congreso sobre el Libro de Texto y Materiales Didácticos, pp. 71-85. Madrid: Universidad Complutense de Madrid.

RICHAUDEAU, F. (1981). Concepción y producción de manuales escolares-guía práctica. Colombia: SECAB-CERLAL - Editorial de la UNESCO.

RIVERA SANTALÓ, L. e IZQUIERDO AYMERICH, M. (1996). Presencia de la realidad y la experimentación en los textos escolares de ciencias. Alambique, 7, pp. 117-122.

RODRÍGUEZ DÍEGUEZ, J. L., ESCUDERO, J. M. y BOLÍVAR, A. (1978). Análisis de estructuras formales del texto escolar. Revista Española de Pedagogía, 140, pp. 73-83.

ROSALES LÓPEZ, C. (1983). Evaluación de textos escolares de primer ciclo de EGB. Enseñanza, 1, pp. 193-208.

SÁNCHEZ HUETE, J. C. (1997). «Análisis de los libros de texto de matemáticas del ciclo medio de la educación general básica». Tesis doctoral. Universidad Complutense de Madrid.

SANGER, M. J. y GREENBOWE, T. J. (1999). An analysis of college chemistry textbooks as sources of misconceptions and errors in electrochemistry. Journal of Chemical Education, 76(6), pp. 853-860.

SANTOS GUERRA, M. A. (1991). ¿Cómo evaluar los materiales? Cuadernos de Pedagogía, 194, pp. 29-31.

SELANDER, S. (1995). Análisis del texto pedagógico (Trad. de A. Sánchez García), en García Mínguez, J. y Beas Miranda, M. (comps.). Libros de texto y construcción de materiales curriculares, pp. 131-161. Granada: Proyecto Sur de Ediciones.

SOLBES, J. (1998). El tiempo y otros nuevos problemas de la enseñanza de la física y la química. Alambique, 18, pp. 107-113.

STRUBE, P. (1989). A content analysis of arguments and explanations presented to students in physical science textbooks: a model and an example. International Journal of Science Education, 11(2), pp. 195-202.

TAMIR, P. y GARCÍA ROVIRA, M. P. (1992). Características de los ejercicios de prácticas de laboratorio incluidos en los libros de texto de ciencias utilizados en Cataluña. Enseñanza de las Ciencias, 10(1), pp. 3-12.

WESTBURY, I. (1990). Textbooks, Textbook Publishers and the Quality of Schooling, en Elliot, D. L. y Woodward, A. (eds.). Textbooks and Schooling in the United States, (pp. 1-22) Chicago: The National Society for the Study of Education. 


\section{ANEXO I}

\section{Ficha inicial}

Editorial:

Año:

Título - Curso:

Autores:

Número de páginas:

Número de unidades didácticas / temas

Número de páginas:

Número de unidades didácticas / temas de química:

Número de páginas:

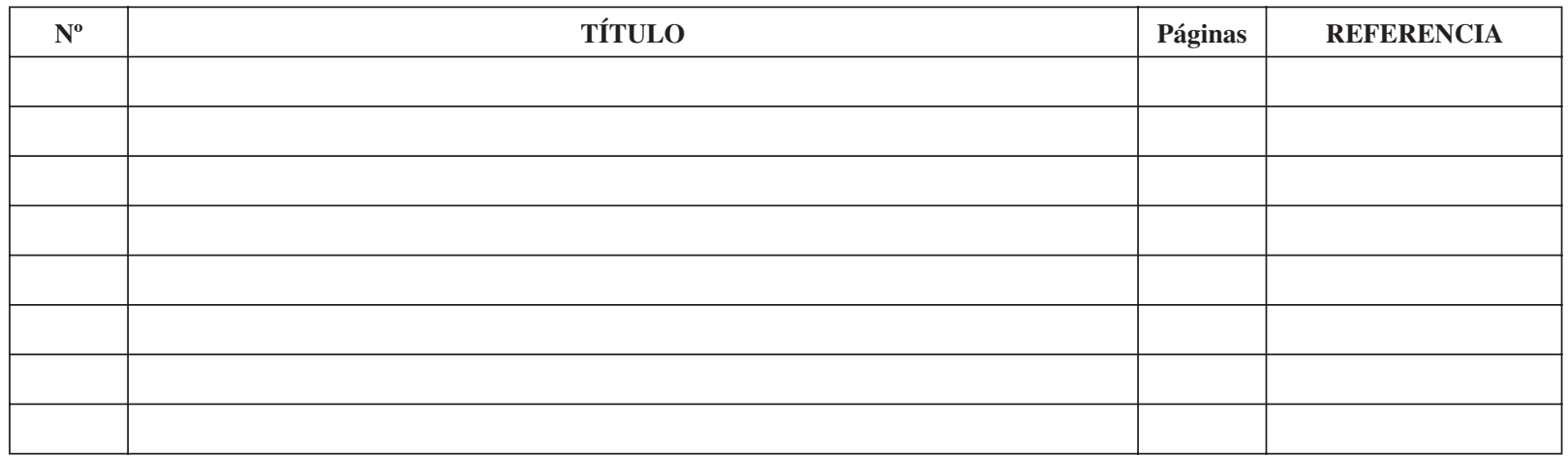

Observaciones:

Plantilla de análisis

REFERENCIA:

1) Estructura de la unidad didáctica / tema:

\begin{tabular}{|c|c|c|}
\hline CLAVE & $\mathbf{N}^{\circ}$, título de la sección y apartados & Extensión \\
\hline & & \\
\hline & & \\
\hline & & \\
\hline & & \\
\hline & & \\
\hline & & \\
\hline
\end{tabular}

2) Para resaltar lo más significativo:

a) Se usan recuadros

b) Se usan letras en negrita

c) Se usan distintos colores

d) Se repite en el margen

e) Otros:

3) Número de actividades de lápiz y papel resueltas: .......

Tipo y código:

a) Cuestiones $[\mathrm{CR}]: \mathrm{n}^{\mathrm{o}}:$.......

b) Ejercicios cualitativos [LR]: $\mathrm{n}^{\mathrm{o}}: \ldots$.

Nombre que les da el libro:

c) Ejercicios cuantitativos [NR]: $n^{\circ}: \ldots$.

Nombre que les da el libro:

Nombre que les da el libro: 
d) Problemas de investigación abiertos [PR ]: $\mathrm{n}^{\mathrm{o}}$ : .........

Nombre que les da el libro:

e) Otras [código]:

Nombre que les da el libro:

Nombre que les da el libro:

Nombre que les da el libro:

Nombre que les da el libro:

d) Problemas de investigación abiertos [PP]: $n^{\circ}$ : ....

e) Otras [código]:

5) Número de actividades experimentales propuestas: ......

a) Experimentos cualitativos [L]: $\mathrm{n}^{\mathrm{o}}: \ldots$.

c) Experimentos cuantitativos $[\mathrm{N}]: \mathrm{n}^{\mathrm{o}}$ : .....

d) Investigaciones [I]: $\mathrm{n}^{\mathrm{o}}: \ldots$.

e) Otras [código]:

Nombre que les da el libro:

Nombre que les da el libro:

Nombre que les da el libro:

6) Número de ilustraciones:

\begin{tabular}{|c|c|c|}
\hline a.1) Fotografías $[\mathrm{F}]$ : & $\mathrm{n}^{\mathrm{o}}: \ldots \ldots \ldots \ldots$ & a.2) Secuencia de fotografías $[\mathrm{SF}]: \mathrm{n}^{\mathrm{o}}$ : \\
\hline b.1) Dibujos [D]: & $\mathrm{n}^{\mathrm{o}}: \ldots \ldots \ldots$ & b.2) Secuencia de dibujos [SD]: \\
\hline 1) Gráficas [G]: & $\mathrm{n}^{\mathrm{o}}: \ldots \ldots \ldots \ldots$ & c.2) Secuencia de gráficas [SG]: \\
\hline d.1) Tablas [T]: & $\mathrm{n}^{\mathrm{o}}: \ldots \ldots \ldots \ldots$ & d.2) Secuencia de tablas [ST]: \\
\hline
\end{tabular}

d.1) Tablas [T]:

$\mathrm{n}^{\mathrm{o}}: \ldots \ldots \ldots . . . .$.

d.2) Secuencia de tablas [ST] :

e.1) Esquemas [E]:

f) Otras [código]:

7) Análisis de los contenidos químicos de cada sección de la unidad didáctica:

\begin{tabular}{|c|c|c|c|c|c|}
\hline CLAVE & CONTENIDOS & $\begin{array}{l}\text { DESARROLLO } \\
\text { TEÓRICO }\end{array}$ & $\begin{array}{l}\text { ACTIVIDADES DE } \\
\text { LÁPIZ Y PAPEL } \\
\text { [tipo-n'] }\end{array}$ & $\begin{array}{c}\text { ACTIVIDADES } \\
\text { EXPERIMENTALES } \\
\text { [tipo-n'] }\end{array}$ & $\begin{array}{c}\text { ILUSTRACIONES } \\
\left.\text { [tipo-n }{ }^{\circ}\right]\end{array}$ \\
\hline & Conceptos & & & & \\
\hline & Procedimientos & & & & \\
\hline & Actitudes & & & & \\
\hline & Conceptos & & & & \\
\hline & Procedimientos & & & & \\
\hline & Actitudes & & & & \\
\hline & Conceptos & & & & \\
\hline & Procedimientos & & & & \\
\hline & Actitudes & & & & \\
\hline & Conceptos & & & & \\
\hline & Procedimientos & & & & \\
\hline & Actitudes & & & & \\
\hline
\end{tabular}

8) En las distintas secciones de la unidad didáctica / tema:

a) Se tienen en cuenta las ideas previas de los alumnos

[clave-tipo- $\mathrm{n}^{\mathrm{o}}$ ]:

.

b) Se establecen analogías con situaciones de la vida cotidiana 
INVESTIGACIŌN DIDÁCTICA

c) Se utilizan referencias históricas

d) Se conecta el tema con otros vistos anteriormente

e) Se tiene en cuenta la interdisciplinariedad

f) Se tienen en cuenta los temas transversales

g)

9) En las actividades de lápiz y papel resueltas:

a) Entre varias opciones se indica la respuesta correcta

b) Se indica si distintas afirmaciones son verdaderas o falsas

c) Se justifica la respuesta

d) Se da más importancia a la utilización de las fórmulas

y al cálculo que al análisis conceptual

e) Se realiza un estudio gráfico

f) Otros:

10) En las actividades de lápiz y papel propuestas se pide que el alumno:

a) Responda preguntas cuya respuesta está explícita en el texto

b) Responda preguntas cuya respuesta está implícita en el texto

c) Elija entre varias opciones la respuesta correcta

d) Indique si distintas afirmaciones son verdaderas o falsas

e) Justifique las respuesta

f) Explique conceptos

g) Cite ejemplos de conceptos

h) Llegue al resultado numérico

i) Analice e interprete el resultado numérico

j) Realice un estudio gráfico

k) Diseñe un experimento

l) Consulte bibliografía

m) Otros:

11) En las actividades experimentales:

a) Se formulan los objetivos

b) Se expone el material necesario en un apartado independiente

c) Se desarrolla paso a paso el procedimiento operativo que debe seguirse

d) Se dan normas de seguridad

e) Se especifica el fundamento teórico de la actividad

f) Aparece una ilustración con el montaje experimental

g) Se especifica el resultado

h) Otros: [clave-tipo- $\mathrm{n}^{\mathrm{o}}$ ]
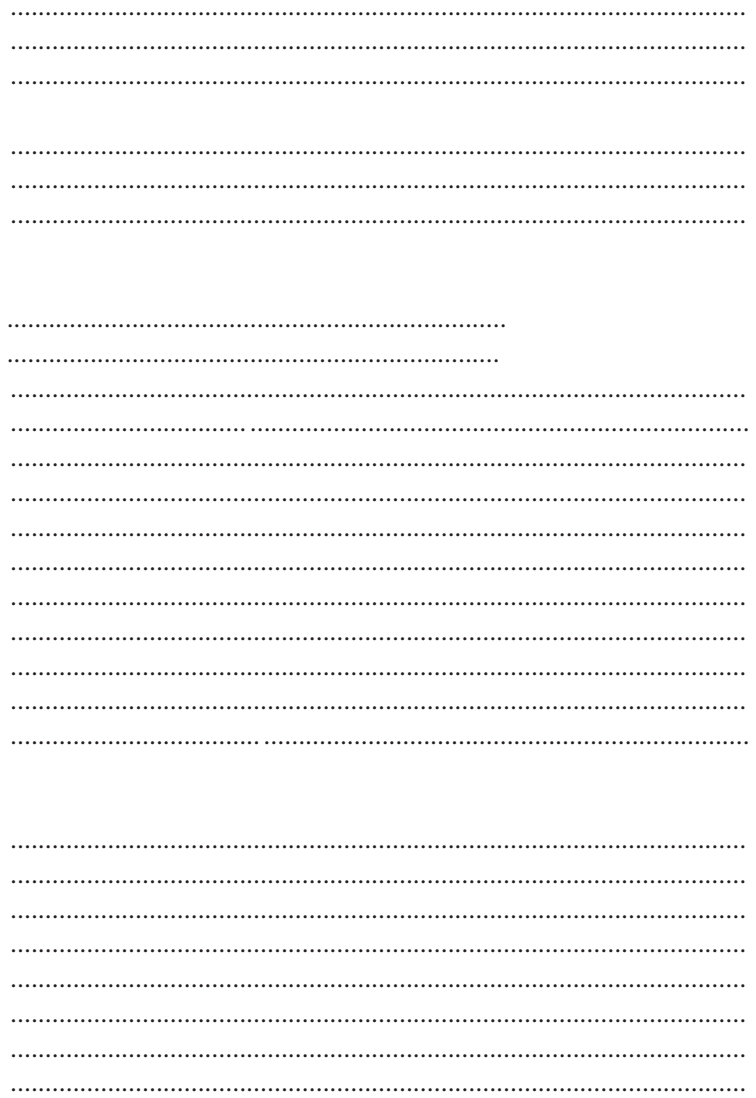
12) En las actividades experimentales propuestas hay que emplear un material:

a) Específico de laboratorio

b) Sencillo, de uso cotidiano

c)Ambos pero intentando que la complejidad sea mínima

13) ¿Se especifica el lugar de realización de la actividad experimental?: NO/ SÍ:

14) En las actividades experimentales se pide que el alumno:

a) Trabaje en grupo

b) Observe lo que ocurre cuando realiza lo que se le indica

c) Tome datos

d) Justifique lo que va sucediendo

e) Realice un estudio gráfico

f) Analice e interprete los resultados

$g$ ) Conteste las preguntas que se le indican $\left(n^{\circ}\right)$

h) Diseñe un nuevo experimento

i) Otros:

15) La función de las fotografías y dibujos es:

a) Facilitar la comprensión de un concepto

b) Plantear una actividad

c) Mostrar un montaje experimental

d) Mostrar la secuencia de una actividad experimental

e) Mostrar científicos ilustres

f) Motivadora / decorativa

g) Otros:

16) Las gráficas, las tablas y los esquemas:

a) Son parte fundamental del desarrollo teórico

b) Son una información añadida pero no necesaria

para la comprensión del texto

c) Repiten una información dada

d) Forman parte de una actividad de lápiz y papel resuelta

e) Forman parte de una actividad de lápiz y papel propuesta

f) Forman parte de una actividad experimental

g) Otros:

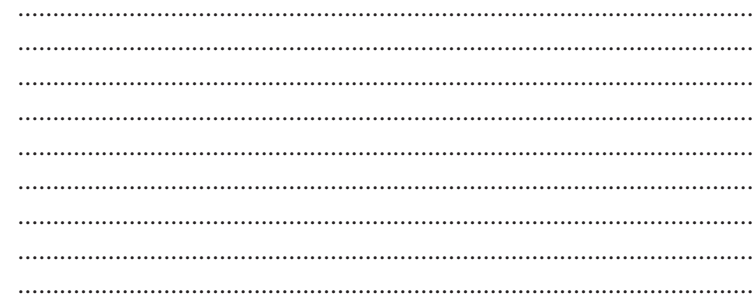

17) Inexactitudes o errores detectados en cada sección de la unidad didáctica :

[clave-tipo]:

18) Observaciones: 


\author{
ANEXO II \\ Legislación española de la enseñanza de química en la educación secundaria obligatoria
}

Ley Orgánica 1/1990 de 3 de octubre (BOE, 4-10-90), de Ordenación General del Sistema Educativo.

Real Decreto 1345/1991, de 6 de septiembre (BOE, 13-9-91), por el que se establece el currículo de la Educación Secundaria Obligatoria.

Resolución de 5 de marzo de 1992 (BOE, 25-3-92), de la Secretaría de Estado de Educación, que regula la elaboración de proyectos curriculares para la Educación Secundaria Obligatoria y establece orientaciones para la distribución de objetivos, contenidos y criterios de evaluación para cada uno de los ciclos.

\title{
Legislación española referente a los libros de texto
}

Real Decreto 388/92, de 15 de abril (BOE, 23-04-92), por el que se regula la supervisión de libros de texto y otros materiales curriculares para las enseñanzas de Régimen General y su uso en los Centros Docentes.

Orden de 2 de junio de 1992 (BOE, 11-6-92), por la que se desarrolla el Real Decreto 388/92, de 15 de abril, sobre supervisión de libros de texto y otros materiales curriculares para las enseñanzas de Régimen General y su uso en los Centros Docentes.

Decreto 1744/1998, de 31 de julio (BOE, 4-09-98), sobre uso y supervisión de libros de texto y demás material curricular correspondientes a las enseñanzas de Régimen General.

Orden de 12 de noviembre de 1992 (BOE, 11-6-92), sobre evaluación de la Educación Secundaria Obligatoria. 